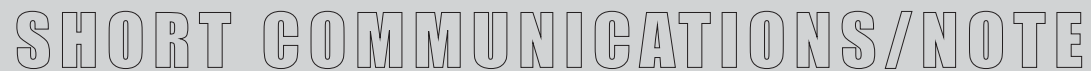

\section{Valutazione comparativa di due metodi automatici per lo screening di Treponema pallidum}

\section{Fulvia Milano, Luigi Cianci, Micaela Pelagi, Loredana Pangaro, Giovanni Olivieri, Paola Verza, Gian Carlo Guazzotti}

\author{
S.O.C. Microbiologia, Ospedale "S. Andrea"ASL-I I, Corso Abbiate 2I, Vercelli
}

Key words: syphilis, chemiluminescence, serology

Comparative evaluation of two automated methods for Treponema pallidum infection screening

\section{SUMMARY}

In the present study we evaluated the performance of two automated chemiluminescence immunoassay for detection of Treponema pallidum antibodies in a routine laboratory approach. One hundred fifty samples, obtained from patients admitted to hospital with no signs or clinical history of syphilis were studied. All samples were tested for Treponema pallidum antibodies by the qualitative method Syphilis TP Architect (Abbott) and with DIASORIN Treponema Screen (DiaSorin) and the reactive results were confirmed by VDRL and IgM ELISA. Western blot IgG and IgM (AID GmbH), VDRL (Abbott) and EIAgen TMPA IgM (Adaltis) were used to analyze discrepant results. One hundred forty four samples had concordant result (96\%) whereas six samples showed discordant results (negative with Architect and positive with DiaSorin).Among discordant tests, 5 positive results obtained with DiaSorin were confirmed by Western blot IgG, while one remained indeterminate. More and extensive evaluations may be useful to define Architect sensibility loss, with homogeneous cohorts of patients including those with a syphilis diagnosis, high risk subjects and patients with potential confounding factors like autoimmune diseases.

\section{INTRODUZIONE}

Oltre che nelle condizioni di sospetto clinico di infezione treponemica attiva, la diagnostica microbiologica per la sifilide trova obbligatorietà in caso di donazione di sangue e d'organo, è fortemente consigliata in gravidanza ed è frequentemente compresa fra gli esami di screening all'atto del ricovero ospedaliero (8).

Nonostante la frequenza di richiesta di diagnostica microbiologica per sifilide rimanga elevata, essa è tuttora gravata da numerose problematiche operative ed interpretative. Treponema pallidum è infatti organismo difficilmente isolabile in coltura, $(3,6)$ ed anche la sua identificazione all'esame microscopico diretto è condizionata dalla presenza di lesioni cutanee in atto al momento dell'accertamento diagnostico; per questo motivo l'indagine sierologia rimane lo strumento diagnostico più accreditato nella routine clinica. Essa si fonda abitualmente su due tipologie di metodiche: quelle non specifiche per il Treponema quali Venereal Disease Research Laboratory (VDRL) e Rapid Plasma Reagine (RPR) e quelle specifiche quali fluorescent treponemal antibodies absorption (FTA-ABS) e Treponema pallidum particle agglutination (TP-PA). I primi due metodi hanno storicamente trovato impiego nei programmi di screening essendo caratterizzati da elevata sensibilità, facilità di esecuzione ed economicità; sono tutta- via gravati da scarsa specificità per reattività nei confronti di antigeni lipoidi di provenienza cellulare e di altre specie non treponemiche, determinando, quando eseguiti in una popolazione a basso rischio, risultati falsamente positivi in una percentuale che sfiora il 50\% (4). Di converso i test treponema-specifici presentano lo svantaggio di una lunghissima persistenza della eventuale positività, fattore che ne pregiudica l'impiego nel follow up dei pazienti. Qualora infatti si studi la cinetica comportamentale degli anticorpi specifici in caso di malattia luetica, anche correttamente trattata, si assiste alla comparsa precoce di IgM durante la fase acuta di malattia con tendenza a scendere nel tempo sotto la soglia di dosabilità, mentre le immunoglobuline di classe IgG permangono in circolo per lungo tempo (a volte decenni), anche in soggetti correttamente trattati. Solo successivamente, infatti, il livello anticorpale tende a decrementare fino alla negativizzazione, rendendo il soggetto potenzialmente suscettibile alle reinfezioni. (5)

A fronte di tali problematiche, la messa a punto di una metodica standardizzabile ed in grado di coniugare affidabilità in termini di risultato con semplificazione gestionale, appare fortemente razionale nel contesto di un laboratorio microbiologico a prevalente valenza assistenziale. A tal fine nel presente studio sono stati valutati compa- 
rativamente due metodi automatici in chemiluminescenza per lo screening di Treponema pallidum, comparandone le performance in termini di sensibilità e specificità, in una popolazione a basso rischio di infezione quali i pazienti ammessi in ospedale con diagnosi non venereologiche.

\section{MATERIALI E METODI}

Nel nostro studio sono stati valutati 150 campioni ematici pervenuti al laboratorio di microbiologia per esami di ruotine peri-ammissione in ospedale. Criterio di esclusione dallo studio era una richiesta di indagine sierologia per lue a fronte di segni e/o sintomi clinici riconducibili ad una possibile infezione in atto e/o recente.

Tutti i sieri sono stati processati con il test qualitativo Sifilide TP Architect della ditta Abbott e contestualmente con analogo test qualitativo LIAISON Treponema Screen della ditta Diasorin. Architect Sifilide TP è un dosaggio immunologico a doppia fase che utilizza la tecnologia CMIA per la rivelazione anticorpale. Nella prima fase vengono dispensate insieme al campione da testare le microparticelle rivestite di antigeni ricombinanti (TpN15, TpN17 e TpN 47) ed il diluente di dosaggio. Gli eventuali anticorpi presenti nel campione si legano agli antigeni presenti sulle microparticelle. Nella seconda fase, dopo il lavaggio, viene aggiunto il coniugato di anticorpi anti IgG e anti IgM umane marcate con acridinio, vengono poi aggiunte, dopo un successivo lavaggio le soluzioni Pre-Trigger (preattivazione) e Trigger (attivazione) che determinano la reazione chemiluminescente misurata in unità di luce relativa (RLU). Esiste una linearità di reazione fra la quantità di anticorpi anti treponema presenti nel campione di saggio e le RLU misurate dal sistema ottico dello strumento. La presenza o l'assenza di anticorpi specifici anti treponema si ottiene dal confronto del segnale chemiluminescente nella reazione con il segnale cutoff determinato da una precedente calibrazione. Qualora il segnale chemiluminescente risulti pari o superiore al cutoff, il campione è considerato reattivo per anticorpi anti treponema.

Il test LIAISON sfrutta invece il principio del dosaggio sandwich ad unico step, con tecnologia di rilevazione in chemiluminescenza CLIA. Antigeni ricombinanti specifici per Treponema rivestono le particelle magnetiche della fase solida e sono legati ad un derivato dell'isoluminolo. Durante l'incubazione, gli eventuali anticorpi presenti nel campione (unitamente a quelli dei calibratori), legano sia la fase solida che l'antigene coniugato, mentre tutto ciò che non è legato viene eliminato nel corso del lavaggio. L'aggiunta alla reazione di reagenti starter induce una risposta in chemiluminescenza misurata da un fotomoltiplicatore con espressione dei risultati in index.

La reattività anticorpale è stata confermata mediante l'impiego di VDRL (Abbott) e con il dosaggio delle immunoglobuline IgM con metodica immunoenzimatica EIAgen TMPA IgM (Adaltis).

Dei 150 sieri quelli con risultato discordante ai due metodi automatizzati oggetto di studio sono stati ulteriormente valutati mediante VDRL, ricerca delle IgM in ELISA e Western blot IgG ed IgM (AID GmbH). Quest'ultimo metodo utilizza proteine naturali (p15.5, p17, p45.5, p47) separate, in base al diverso peso molecolare, mediante SDS-PAGE elettroforesi e successivamente trasferite su membrana di nitrocellulosa. Dopo incubazione con "blocking buffer", per reidratare e bloccare i siti liberi ed evitare così legami non specifici, le strisce sono incubate con i sieri prediluiti permettendo agli anticorpi anti treponema presenti di legarsi agli antigeni blottati. I passaggi successivi, dopo gli opportuni lavaggi, sono quelli di una reazione immunoenzimatica classica. L'avvenuto legame antigene-anticorpo è evidenziato sulla striscia come banda di colore blu.

\section{RISULTATI}

La valutazione comparativa dei risultati ottenuti con le due metodiche qualitative in chemiluminescenza ha fornito risultati concordanti per 144 campioni (96\%): 136 concordanti negativi e 8 concordanti positivi. In 6 campioni (4\%) si è invece evidenziato un risultato discordante, con assenza di reattività al Sifilide TP Architect rispetto alla reattività identificata dal metodo LIAISON Treponema Screen (tabella 1).

Valutando i 6 campioni discordanti con test di conferma (Western blot IgG e IgM, VDRL ed ELISA IgM), la reattività identificata dal LIAISON Treponema Screen ha trovato conferma con immunoblotting IgG, ma sia la VRDL che la ricerca degli anticorpi IgM con Western blot e metodo ELISA hanno dato esito negativo, permettendo così di escludere un'infezione luetica in atto e/o recente in tutti e sei i campioni (tabella 2).

Nella tabella 3 vengono riportate per tutti i singoli campioni risultati reattivi la risposta alle singole proteine blottate, con il corrispondente criterio interpretativo desunto dalla metodica Western blot AID, dal quale si evince come 5 campioni (AE) siano classificabili come positivi, mentre per un campione $(\mathrm{F})$, il risultato debba essere interpretato come dubbio.

A tal proposito, nelle figure I e II vengono riportati come esemplificazione di campione rispettivamente positivo e dubbio le Western blot IgG dei campioni $\mathrm{D}$ ed $\mathrm{F}$. 
Tabella I. Confronto LIAISON Treponema Screen vs. ARCHITECT Sifilide TP

\begin{tabular}{lcc}
\hline & Non reattivi & Reattivi \\
\hline Campioni Concordanti & 136 & 8 \\
\hline Campioni Discordanti & 0 & 6 \\
\hline \multicolumn{2}{c}{ concordanza $96 \%$} \\
\hline
\end{tabular}

Tabella 2. Dettaglio dei 6 campioni discordanti con LIAISON Treponema Screen vs. ARCHITECT Sifilide TP

\begin{tabular}{cccc}
\hline & Positivo & Dubbio & Negativo \\
\hline Western Blot IgG & 5 & I & \\
\hline Western Blot IgM & & & 6 \\
\hline ELISA IgM & & & 6 \\
\hline VDRL & & 6 \\
\hline
\end{tabular}

Tabella 3. Western Blot lgG

\begin{tabular}{lll}
\hline Campione & Bande reattive & \\
\hline Campione A & pl5.5 - p45.5 & deb. Positivo \\
\hline Campione B & pl5.5 - p47 & Positivo \\
\hline Campione C & pl5.5 - p47 & Positivo \\
\hline Campione D & pl7 - p47 & Positivo \\
\hline Campione E & p47 - pl5.5 & Positivo \\
\hline Campione F & pl7 & Dubbio \\
\hline
\end{tabular}

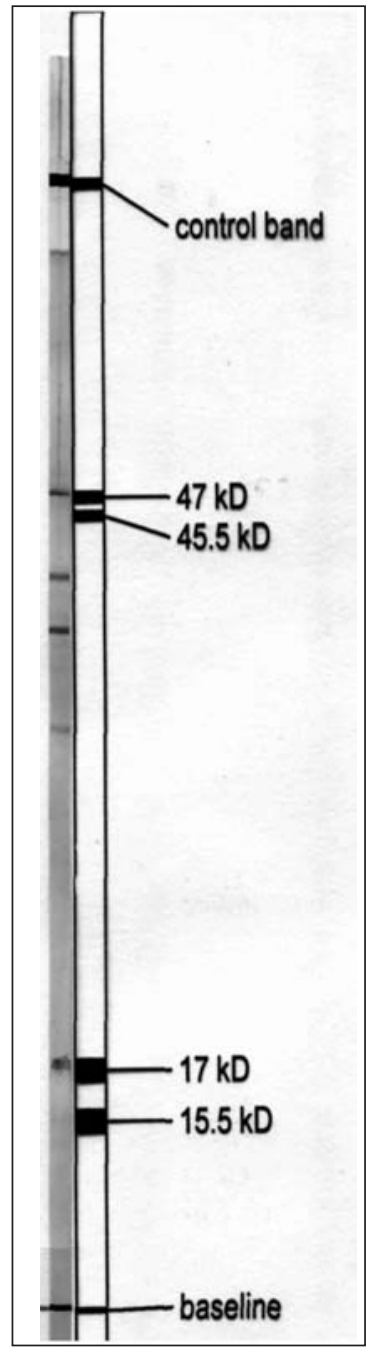

Figura I. (campione D)

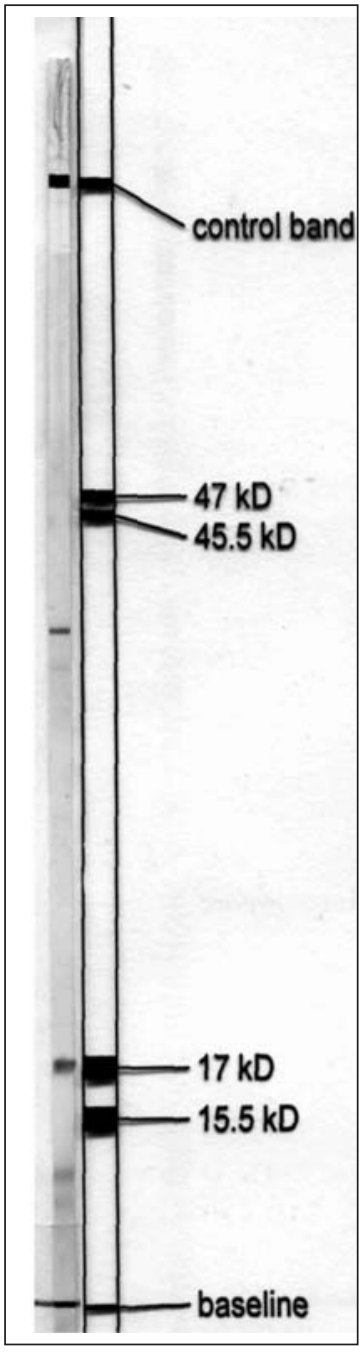

Figura II. (campione F)

\section{DISCUSSIONE}

Nello studio della patologia luetica, oltre che alla valutazione dei segni e sintomi suggestivi di malattia, peraltro utilizzabili solo nelle fasi precoci o acute della stessa, si fa abitualmente ricorso all'indagine sierologica, che grazie alla possibilità di dosare anticorpi anti treponema specifici, consente lo studio di tutte le fasi che caratterizzano l'evoluzione della sifilide, ivi comprese le forme quiescenti (5).

La lue rimane un problema di significativo interesse clinico ed epidemiologico, per cui, in assenza di sostanziali possibilità di diagnosi diretta, le tecniche indirette rimangono il cardine dell'approccio diagnostico. Nel tentativo di superare le problematiche di sensibilità e specificità dei metodi tradizionali, sono stati proposti diversi metodi alternativi. Di particolare interesse appaiono le metodiche che utilizzano antigeni ricombinanti anti treponema differenti le cui performance sono state puntuale oggetto di valutazioni comparative $(1,7,9)$. In particolare la tecnologia della chemiluminescenza appare di sicuro interesse per l'elevata sensibilità e specificità oltre che per la possibilità di completa automazione. I test in chemioluminescenza, quindi possono verosimilmente essere proposti come potenziale test di screening ideale ovvero come supporto ideale ai test tradizionali. (5)

Nella nostra esperienza, entrambi i metodi diagnostici basati sulla tecnologia in chemiluminescenza messi a confronto si accomunano per l'ottima maneggevolezza in termini di automazione oltre che per i costi contenuti; tuttavia, è necessaria una seria riflessione sulla evidente perdita di sensibilità del metodo Sifilide TP Architect rispetto al metodo LIAISON Treponema.

In questa ottica si impone come prioritaria la necessità di un'attenta disamina degli antigeni utilizzati dai due test nel rilevare la risposta anticorpale, oltre che sul principio operativo dei metodi stessi. Si deve, invero, rimarcare come i falsi negativi rilevati con il metodo Sifilide TP Architect non fossero riferiti ad infezione attiva, in quanto le indagini sierologiche per la ricerca delle IgM specifiche, basate su metodi e principi differenti, hanno infatti dato esito negativo. Il falso negativo non avrebbe quindi pregiudicato la eventuale gestione terapeutica, tuttavia la sua frequenza non appare casuale, rendendo tale test forse meno idoneo del comparatore ad indagini di screening, dove è prioritario garantire sensibilità massimale.

La nostra indagine comparativa di metodi diagnostici di screening ha nel basso numero di campioni esaminati un evidente limite; tuttavia riteniamo che essa sia sufficientemente esaustiva di quanto 
siano necessari ulteriori studi di validazione, verificando la qualità dei test su campioni di maggiori dimensioni, su popolazioni con differente incidenza di fattori di rischio, su pazienti con fondato sospetto clinico di malattia attiva ed infine su pazienti con elevato rischio di falsi positivi quali i portatori di patologie autoimmuni.

Con riferimento infine ai test di conferma, senza dubbio la metodica Western blot ha dimostrato, anche sulla base di quanto segnalato in letteratura (2), eccellenti performance tanto in termini di sensibilità (attribuibile verosimilmente alla tipologia di antigeni utilizzati) che di specificità (2). Questo test, anche sulla base della nostra, sia pur limitata casistica, può sicuramente essere riconosciuto come valida alternativa all'impiego di associazioni diagnostiche differenti per la conferma sierologia dell'infezione luetica.

\section{BIBLIOGRAFIA}

1. Castro R, Prieto ES, Santo I, Azevedo J, da L. Esposto F. Evaluation of an enzyme immunoassay tecnique for detection of antibodies against Treponema pallidum. J. Clin. Microbiol., 2003; 41: 250-3.

2. Ebel A, Vanneste L, Cardinaeles M, et al. Validation of the INNO-LIA syphilis kit as a confirmatory assay for Treponema pallidum antibodies. J. Clin. Microbiol., 2000; 38: 215-19

3. Fieldsteel AH, Cox DL, Moeckli RA. Infect. Immun., 1981; 32: 908-15.

4. Larsen AS. Syphilis. Clin. Lab. Med., 1989, 9: 545-57.

5. Marangoni A, Sambri V, Accardo S, et al. Evaluation of LIAISON treponema screen, a novel recombinant antigen-based chemiluminescence immunoassay for laboratory diagnosis of syphilis. Clin. Diagn. Lab. Immunol., 2005; 12: 1231-4.

6. Norris SJ. In vitro cultivation of Treponema pallidum: independent confirmation. Infect. Immun., 1982; 36: 437-9.

7. Sambri V, Marangoni A, Eyer C, et al. Western immunoblotting with five Treponema pallidum recombinant antigens for serological diagnosis of syphilis. Clin. Diagn. Lab. Immunol., 2001 ; 8: 534-9.

8. Young H., Syphilis: serology. Dermatol. Clin., 1998; 16: 691-8.

9. Zrein M, Maure I, Bousier F, Soufflet L, Recombinant antigen-based enzyme immunoassay for screening of Treponema pallidum antibodies in blood bank routine. J. Clin. Microbiol., 1995; 33: 525-7.

Fulvia Milano

S.O.C. Microbiologia

Ospedale "S.Andrea" ASL 11

Corso Abbiate 21, 13100 Vercelli

Tel.: 0161 593727; Fax: 0161593501

E-mail : fulvia.milano@asl11.piemonte.it 